Article

\title{
What is Interdisciplinarity in Practice? Critical Reflections on Doing Mobility Research in an Intended Interdisciplinary Doctoral Research Group
}

\author{
Dominic Villeneuve *(1), David Durán-Rodas, Anthony Ferri, Tobias Kuttler, Julie Magelund, \\ Michael Mögele, Luca Nitschke ${ }^{\circledR}$, Eriketti Servou ${ }^{\circledR}$ and Cat Silva \\ Chair of Urban Structure and Transport Planning, TUM Department of Civil, Geo and Environmental \\ Engineering, Technical University of Munich, 80333 Munich, Germany; david.duran@tum.de (D.D.-R.); \\ anthony.ferri@tum.de (A.F.); tobias.kuttler@tum.de (T.K.); julie.magelund@tum.de (J.M.); \\ michael.moegele@tum.de (M.M.); luca.nitschke@tum.de (L.N.); eriketti.servou@tum.de (E.S.); \\ cat.silva@tum.de (C.S.) \\ * Correspondence: dominic.villeneuve@tum.de; Tel.: +49-89-289-10455
}

Received: 30 July 2019; Accepted: 23 December 2019; Published: 25 December 2019

\begin{abstract}
Lately, there has been a tendency in academia to call for more interdisciplinary research on sustainable mobility. However, there is a lack of empirical research on practiced interdisciplinarity. This paper seeks to address this by exploring the practices of an intended interdisciplinary doctoral research group. Specifically, it presents the study of a collaborative autoethnography using individual vignettes and qualitative data analysis. The results classify the identified interdisciplinary practices into three main categories: Interactions, productive processes, and negotiation processes, where interactions serve as a carrier for negotiation and productive processes. This also uncovers advantages and challenges associated with these interactions. Furthermore, the analysis reveals intersubjectivity as an important component of the infrastructure of interdisciplinarity involved in both processes. Finally, we call for a reevaluation of the hierarchical thinking about the different levels of interdisciplinarity, going from disciplinary to multidisciplinary to interdisciplinary to transdisciplinary research. We conclude that for interdisciplinarity to happen in practice, it requires having a combination of various disciplines, ontologies, and a common "wicked" problem to solve. We also find that developing an interdisciplinary research environment requires researchers to embark on a shared journey of reaching a higher level of intersubjectivity through continuous interactions and discussions, while also negotiating conflicts.
\end{abstract}

Keywords: research practice; interdisciplinary practice; intended interdisciplinarity; reflexive methods; doctoral research; sustainable mobility; interdisciplinary research; intersubjectivity in research; autoethnographic vignettes; collaborative autoethnography

\section{Introduction}

Mobility and transportation are gaining more importance in current debates about (un)sustainability in our existing society and everyday practices and its consequences for future generations. Therefore, a vast set of actors including politicians, non-governmental organizations (NGOs), companies, and researchers, in particular, argues for a profound shift towards a sustainable mobility system [1-4]. Global socio-environmental issues like climate change and (un)sustainability are often described as "wicked problems" (see [5]) that are very difficult to define due to their interrelation with an indefinite number of other problems. Thus, it is impossible to just "solve" the problem, 
especially within a single discipline. Nothing less than collaborating, combining, and integrating different (non)scientific perspectives and knowledge is needed to identify and tackle the problem (see [6-8]). Moreover, inter- and transdisciplinary research formats seem suitable for scientific inquiry on sustainability-related issues (e.g., see $[9,10])$ and particularly for addressing mobility-related issues (e.g., see [11-14]). Such an integrative view on mobility has become increasingly prominent in mobility research through interlinking immaterial (meanings, perceptions, etc.) and material elements of mobility. The interdisciplinary view on mobility was introduced by John Urry [15] and later established as The New Mobilities Paradigm by Sheller \& Urry [11]. The history of mobilities research shows that the phenomenon has been further researched from a variety of disciplines and often with intended interdisciplinarity, meaning that the research is carried out within interdisciplinary projects and between researchers with different disciplinary backgrounds [11,15-20]. However, few publications have directly addressed the relation between mobility research and interdisciplinarity specifically.

\subsection{Problem Statement}

While there is research using an interdisciplinary approach to the sustainable mobility problem, there is a lack of reflection on the practice of interdisciplinary research in this field (nonetheless, see Weingart and Stehr [21] for a contribution specifically focusing on practicing interdisciplinarity). An exception to this is Maasen [22], who reflects on how interdisciplinarity is practiced within a research group at the Center for Interdisciplinary Research (ZiF) in Bielefeld, Germany, and critically describes practiced interdisciplinarity as "inducing some interactions at some intersections of some inter-fields" [22]. However, Maasen writes from an individual reflexive point of view, reflecting the voice of the single researcher as part of an interdisciplinary group without giving voice to the other members of the group or the group as a collective. Since interdisciplinary research generally involves multiple scientists and, in contrast, this paper seeks to show the point of view of the group (the eight Hans-Boeckler-Stiftung (HBS)-funded PhD candidates of the mobil.LAB and their postdoc coordinator) and the interactions and characteristics occurring in our everyday work.

To investigate our interdisciplinary research group as a whole, considering each of our respective experiences, we carried out a collaborative autoethnography using individual vignettes and collective qualitative data analysis. Although the vignettes, which are the main empirical data for this paper, are written from an individual point of view, the data analysis of the vignettes was carried out as a group, and the paper itself was written as a group contribution. In attempting to write a reflexive paper as an interdisciplinary research group, we aim to give a voice to all members, as well as establish the group as a collective. By reflecting on interdisciplinarity from the point of view of a group, our contribution highlights the importance of not only interdisciplinarity itself, but also interdisciplinarity in practice.

As outlined above, working with an interdisciplinary approach often comes out of a wish to address the "wicked problem" of sustainability and mobility; nevertheless, the manner in which this is actually practiced in research groups like the mobil.LAB remains, at best, vague. Therefore, this paper aims to address how an intended interdisciplinary set-up works in practice, by describing the everyday interactions and understanding our interdisciplinary practices.

\subsection{The mobil.LAB Doctoral Research Group}

The call for interdisciplinary mobility research to overcome unsustainable mobility cultures-outlined above - and its societal urgency prepared the ground for the mobil.LAB doctoral research group funded by the foundation of the confederation of German trade unions (DGB), Hans-Boeckler-Stiftung (HBS).

Based on the ideals of interdisciplinarity and influenced by cultural and mobility studies [23], the creation of the second phase of the mobil.LAB doctoral research group, consisting of eight doctoral candidates at the Technical University of Munich (TUM) and Nürtingen-Geislingen University of Applied Sciences (HfWU), is intended to "shape mobility cultures". The purpose for using the already existing concept of mobility cultures [24] for the mobil.LAB as a "guiding principle for future 
research" [25] was to derive a number of research prerequisites for "formulating an approach to an interdisciplinary mobility theory" [25] and to help guide every doctoral candidate's individually defined research in one way or another.

The first phase of the mobil.LAB, which ran from 2011-2016 at the Technical University of Munich (TUM), focused largely on the theme of sustainable mobility. Through the research which was carried out during this phase, the mobil.LAB developed to "become a place where research into mobility, no longer simply transport planning, is carried out" [23]. This research thus established the ground for the second and current phase of the mobil.LAB, in which the doctoral candidates began their research between October 2016 and March 2018, under the common research theme of "Shaping Mobility Cultures".

In general, the mobil.LAB focuses on the complexities of mobilities research in the Munich region and abroad. Each individual research topic from the doctoral candidates encompasses ideas and methodologies from a variety of disciplines, and it is intended that the interdisciplinary set-up of the group and its activities will enable the production of eight highly useful doctoral dissertations on various aspects of sustainable mobility. Typically, the research and methods have foundational elements of the individual researchers' disciplinary backgrounds, ranging from engineering and natural sciences, social sciences, to design. The research group is also comprised of one postdoc coordinator, four associated doctoral candidates, two co-speakers, and ten affiliated professors from various universities. Thus, our research group navigates through a complex environment, which actively engages with a number of doctoral candidates and professors from other universities and departments, who are not funded by the HBS.

The members of the mobil.LAB also interact and participate in a variety of events and projects throughout the year in both everyday working environments as well as several social platforms. Since the group is a collection of academics from various departments and universities, the traditional set-up of the day-to-day activities is different for each individual member. For example, the eight mobil.LAB doctoral candidates and the postdoc coordinator funded by the HBS meet on a daily basis in an office environment and meet once a month for a research colloquium, a check-in meeting, and a $\mathrm{PhD}$ clinic. The group also meets through self-organized platforms, such as a monthly reading group. These events sometimes include the associated doctoral candidates who are part of the mobil.LAB but are not funded by the HBS, as well as the many professors and supervisors affiliated with the group.

The mobil.LAB colloquium is an opportunity for a doctoral candidate to present their research to the larger group and to lead the discussion and debate about their research-often focusing on particular aspects of the research the candidate is working through or has just completed. As this monthly meeting is the main platform where all members are present, the goal of this particular event is to help foster a sense of community and to provide an opportunity for both formal and informal discussions surrounding the presenter's individual research topic, in relation to each individual's experiences. The other mobil.LAB events, such as the $\mathrm{PhD}$ clinic and check-in meetings, are solely for the doctoral candidates to ask each other for advice in a supportive environment. The check-in meeting consists of a round-table status update by each candidate and discussions relating to the day-to-day work environment. The $\mathrm{PhD}$ clinic is a gathering where a few candidates present a problem they are facing or a question they need help to investigate, which is then discussed as a group in an attempt to resolve the particular issue. Events, such as the now no longer existing reading group, are examples of the mobil.LAB doctoral candidates self-organizing to create opportunities for research collaboration and communication. The external events, which are far less frequent than the internal events, allow opportunities for the members of the mobil.LAB to connect with researchers from abroad, as well as provide opportunities for group reflection and planning; for example, at the annual mobil.LAB retreat.

These socio-spatial platforms used by the researchers to communicate and interact with one another provide ample opportunity for inter- and multidisciplinary interaction. It is these specific interactions upon which this paper is empirically based. The remainder of this paper presents a reflexive empirical inquiry of the everyday interactions in an intended interdisciplinary doctoral research group. First, we provide a brief literature review including the conceptual framework 
that was used. In Section 3, we present an overview of our methodology in doing a reflexive investigation of our everyday work by using collaborative autoethnography. Here, we discuss our use of autobiographical vignettes and a qualitative analysis in combination with reflexive elements-such as collective qualitative data analysis (QDA), round-table discussions, and interpretation of the generated empirical material-in order to provide insights into unconscious and hidden practices in our everyday work. Building on this, we present the empirical findings in Section 4, followed by a discussion in Section 5 and a conclusion in Section 6. In the discussion, we aim to show how intended interdisciplinarity does not create interdisciplinarity itself, but rather that interdisciplinarity is the product of continuous work within a group, and that intersubjectivity, reflection, and discussions are necessary in order to create, but also uphold, interdisciplinarity.

\section{Literature Review and Conceptual Framework}

\subsection{Disciplines and Interdisciplinary Research}

In this paper, we understand disciplines as "[ ... ] institutions that coordinate the production of knowledge [ ... featuring ... ] a set of instructions on how to generate knowledge including subject definitions, conceptual approaches, cognitive structures, goals, and norms" [26] cited in [27]. Interdisciplinary research has become more and more prevalent $[28,29]$. It has especially been called for in research dealing with sustainable mobility which involves environmental, social, and economic components [30]. At a basic level, we define interdisciplinary research as research benefiting from the knowledge and expertise of more than one scientific discipline. Castán Broto, Gislason, and Ehlers refer to it as "research that cuts across disciplines" [27]. Aboelela et al. [31] provide an exhaustive definition based on a systematic literature review and interviews with interdisciplinary researchers: "Interdisciplinary research is any study or group of studies undertaken by scholars from two or more distinct scientific disciplines. The research is based upon a conceptual model that links or integrates theoretical frameworks from those disciplines, uses study design and methodology that is not limited to any one field, and requires the use of perspectives and skills of the involved disciplines throughout multiple phases of the research process".

Groups of researchers from different disciplines can play with the boundaries between the disciplines in interdisciplinary work [26]. However, as Rau and Fahy [9] note, a lack of agreement between researchers from social sciences and natural sciences can be an important barrier to trustworthy and well-integrated sustainability research. For example, "Different theoretical traditions in the social sciences are often underpinned by different and perhaps incompatible views of the human condition, including fundamental divergences with regard to the degree of rationality ascribed to human social (inter)action, the importance of societal structures vis-à-vis agency, or the role of the individual within social organizations and institutions" [9].

Interdisciplinary research has been conceptualized in a ladder with different levels of interdisciplinarity, going from disciplinary to multidisciplinary to interdisciplinary to transdisciplinary—see [32] and [33] cited in [31]. For Lattuca [34], “[ .. ] interdisciplinarity exists on a continuum [from] the informal communication of ideas, such as might occur in a conversation between colleagues from different disciplines, [to] formal collaboration, such as research or teaching teams comprised of faculty from different disciplines".

\subsection{Mobility Cultures as an Interdisciplinary Research Framework}

Contemporary mobility researchers and advocates for sustainable transport alike strongly argue for significant shifts in mobility cultures in order to overcome unsustainable mobility practices. In a German context of interdisciplinary mobility research, Deffner et al. [24] started to work with a research framework focusing on "mobility cultures" incorporating a vast set of "subjective" and "objective" mobility-related aspects. Here, bringing both spheres of transportation scholars (e.g., planning, infrastructure, built environment) and social sciences (e.g., political discourses, lifestyles) into play 
for researching mobility cultures aims for an interdisciplinary research approach addressing mobility. Building on the concept of mobility cultures, a number of studies started to identify (urban) mobility cultures and their characteristics [35-39].

On the one hand, the aforementioned studies apply the concept of mobility cultures for researching different kinds of mobility cultures. On the other hand, the concept was designed for the "development of $\mathrm{a}[\mathrm{n}]$ integrated concept for planning, communication, and implementation of a sustainable multi-optional mobility culture" [24], which can also be applied for setting up research for groups like ours-the mobil.LAB.

\section{Research Design and Methods}

The research for this article is a reflective and reflexive exercise by the members of the mobil.LAB on the members of the mobil.LAB, and therefore classifies as autoethnography. Autoethnography is a type of research that analyzes the researcher and their experience for understanding cultural experience $[40,41]$. It thereby is grounded in an inherently subjective understanding of research, favoring reflexive approaches to research [42].

However, a "classical" autoethnography would be undertaken by one individual researcher, whereas in our case, it was carried out as a collaborative autoethnography using "personal stories as windows to the world [to] interpret how [our] selves are connected to [our] sociocultural contexts and how the contexts give meanings to [our] experiences and perspectives" [43]. We collaboratively collected and analyzed autobiographical data in order to "gain a meaningful understanding of sociocultural phenomena reflected in [our] autobiographical data" [43]. Through this, we intended to elicit a deeper and broader understanding of what interdisciplinarity means in practice.

Collaborative autoethnography [43] allows us to deeply tap into the richest source of data on the influences of working in an intended interdisciplinary group-ourselves as members of such a group. Through our collective meaning-making of our individual and shared research practices, we sought to critically investigate our autobiographies in relation to the intended interdisciplinary set-up of our sustainable mobility research group. The collaborative approach helps to escape an overly self-obsessed account, of which autoethnographic research is sometimes accused; however, it bears the problem of finding consensus while avoiding the silencing of certain perspectives [43,44]. As forms of data generation, we chose autobiographical self-reflective vignettes, a common method of ethnographic research that is sometimes used in autoethnographic research $[45,46]$ and combined these with a collaborative QDA (for examples of research groups using a similar collaborative autoethnographic approach, see [47-51]). At several stages of our research, the whole group discussed the ongoing research process and analysis, thereby alternating between individual and collective ways of sense- and meaning-making. We practiced an inclusive atmosphere within the discussions, particularly paying attention to the representation and understanding of the whole group.

The research design follows a four-step methodology using the approach referred to as the "concurrent model" [43], which is presented in Figure 1 and discussed in detail below.

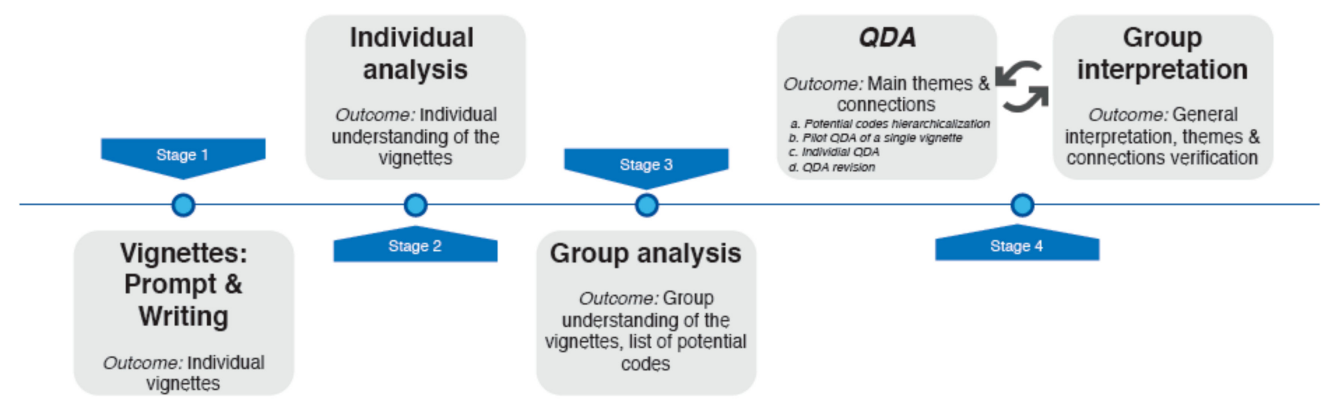

Figure 1. Flowchart depicting the research design. 
In the first stage, to prepare for writing their vignettes, the group reflected on their disciplinary backgrounds and the presence of other disciplines in the group. Figure 2 shows a word cloud of all the disciplines regularly interacting within the group (this includes the backgrounds of all nine coauthors, the four associated doctoral candidates, and one professor; nine people associate to social sciences and five to natural sciences). The figure also shows the important presence of various social sciences, with urban planning as the most frequent discipline, followed by sociology and two interdisciplinary fields, namely mobilities research and urban studies.

Each author reflected and noted interdisciplinary interactions during their day-to-day work and then wrote their own reflexive vignette. The group also discussed the use of vignettes and looked at sample vignettes. An inspirational prompt for the vignettes was formulated for a common understanding of the task, presenting the general principles that every author should follow to write their reflexive narratives. The prompt was developed by two of the PhDs after a group discussion on the method to be used and general aspects of what interdisciplinarity is composed of (e.g., communication between researchers, ontology). The prompt presented three parts: (1) The main question to focus on: "How does the interdisciplinary set-up of the mobil.LAB influence your individual research on mobility and (re)shape the predefined common research theme of mobility culture?", (2) practicalities (e.g., words limit of 750-1000 words, written in the first person), and (3) a list of potential points of which to be reflexive, including the following aspects:

- Insights into the interrelation of the interdisciplinary set-up and daily routines, and interactions within the group and the general research environment.

- Interactions influencing the individual research projects, including ontology, argument building, communication, and methodology.

- Critical reflection on what interdisciplinarity in practice is.

- Recommended activities to be reflexive of: Workshops, colloquia, group activities, social activities, $\mathrm{PhD}$ clinics, retreats, reading groups, daily interactions in the office, activities outside the mobil.LAB which we felt we were influenced by the mobil.LAB, moments when talking about the mobil.LAB (outside of the mobil.LAB), or anything that came to our minds and that we thought is relevant.

Vignettes were produced by all of the HBS-funded PhD candidates of the mobil.LAB who share workspaces together and the postdoc coordinator of the group (the nine coauthors of this paper). Although the styles of the vignettes varied for each individual, we find that they generally tended to include both accounts that were already reflected and rationalized after the fact, as well as more emotional and in situ feelings (see [52] for an example of vignette-based research using a combination of vignette styles).

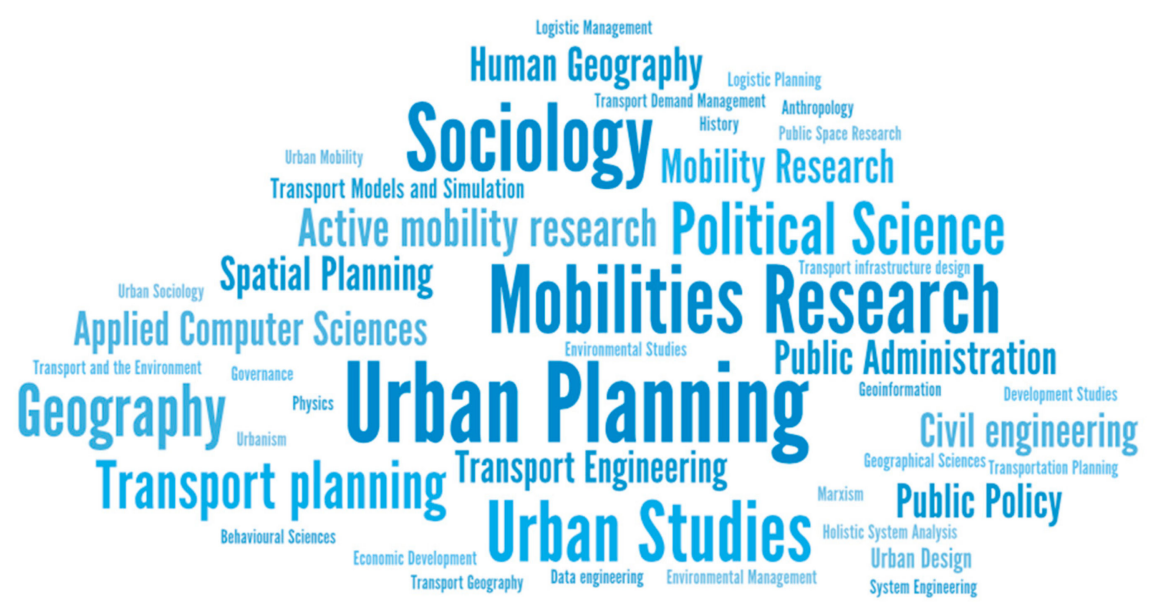

Figure 2. Word cloud of the disciplinary fields within the research group (the size is correlated with the frequency of each disciplinary field). 
We analyzed the vignettes in two stages, the second and third stages of the methodological process. In the second stage, we individually read each vignette, paying attention to similarities and differences in order to get to know our autobiographical material. Then, in the third stage, we held a workshop to collectively discuss the similarities and differences to develop a common understanding of driving themes. This workshop was supported by a written comment from one of the researchers who couldn't attend the meeting due to a research stay. The outcome of this workshop was a list of codes that served as a starting point for the QDA conducted in the fourth research stage.

The QDA was performed by a team of four researchers from the group who had previous experience with qualitative data analysis in their own research. The software MaxQDA was used for the analysis. A coding structure was created in an iterative process. Together, the QDA team set up the coding tree developed by the whole group during the vignette analysis stage. The QDA team then coded one of the vignettes so that they could familiarize themselves with the codes and confirm the appropriateness of the coding. The initial coding scheme was further refined through this process, and the codes were hierarchized. The vignettes were then randomly assigned to coders while ensuring that no one would have to analyze their own individual vignette.

The coding was done sequentially and memos were used to document questions or issues that arose during the coding. As suggested by Patton [53] cited in Ryan and Bernard [54], we used triangulation through multiple analysts as a form of quality assurance and calibration of our usage of the codes. As described by Lincoln and Guba [55] cited in Barbour [56], each researcher examined the coding of another researcher to evaluate it and identify discrepancies. The QDA team convened to discuss the variations with the coding and solved them together. Then, the most common codes, as well as those identified as priorities during the initial group analysis meeting, were investigated further as a group, following a procedure akin to the process described by Gibbs [57]. This process consists of retrieving the quotes from each code and reviewing them together, looking for intersecting or sequentially used codes to identify relationships between the various themes represented. This analysis process started with the most commonly used codes signifying the main themes. New meta-codes were added to identify the advantages and challenges discussed inside the other codes. Finally, the QDA team presented their analysis to the whole group. These results and their interpretations were thoroughly discussed and adjusted based on the coauthors' feedback.

\section{Results}

In our empirical material, the nine individual vignettes, the researchers reflected mostly on their everyday work as individuals, within the group, and how this has influenced us and the group dynamics. One topic that was part of the vignette prompt, the reflection on our common theme of "shaping mobility cultures", was only treated implicitly and not by all members. Therefore, the results focus on our everyday work, our interactions, and their attending discussions. The results revolve around three main categories, which emerged from grouping together the codes and analyzing their intersections and interconnections, namely (1) interactions, (2) productive processes, and (3) negotiation processes. The interactions of different kinds thereby form the basis and carrier for the productive and negotiation processes to take place.

\subsection{Interdisciplinary Interactions}

One of the three main categories that emerged from the data set is "interactions". Interactions are the basis and the frame through which the intended interdisciplinarity of the group plays out in everyday life. The interaction in the group takes place in a variety of spatiotemporal platforms; namely, everyday working, the monthly colloquium, the monthly $\mathrm{PhD}$ clinic, and the no-longer-running monthly reading group. The interactions resemble an interactive system enabled through these spatiotemporal platforms and comprised of inputs, means of communication, and dynamics, resulting in consequences described in the "productive process" and "negotiation process" sections below. 
The aforementioned input is divided into two levels-more specifically, individual input and group input. The individual input refers to the diversity of worldviews and ontologies, mentalities, disciplinary backgrounds, and research approaches (ranging from qualitative to quantitative) that each of the individual researchers brings into the group. This diverse individual input sets the stage for viewing one's research from different angles, generating new ideas and reflections, and going deeper into methods, theory, and argument building. Thus, this gives the individual researchers "a chance to further analyze [their] own direction, and also allows [them] to defend the way [they] would like to proceed" (Vignette 1). This usually happens at the formal platforms of the group, such as the monthly colloquium or the PhD clinic. In contrast, when it comes to everyday working, it appears that it is more likely for people with similar approaches (e.g., qualitative) and disciplinary backgrounds to discuss their work with each other in their everyday informal interactions in the office, as they can go deeper into theoretical and methodological questions. The exchange of feedback between people with different research approaches in everyday working settings is less common.

The group input refers to the collective contributions emerging from the social life, the notion of family (or friendship), and the combination of different perceptions within the group. The social life seems to have created a safe and open environment for both work and personal discussions as well as opinion exchange in the group, which some of the members relate symbolically to the notion of family, while others relate it more to the notion of friendship. This interplay between personal and work aspects builds up a support network; as one of the group members puts it: "We are encouraged to discuss with each other our doubts and concerns, which is highly beneficial, as it gives us a sense of a larger support network" (Vignette 1). The combination of different perceptions on a common topic in the group might often lead to problem solving, but at the same time, it often creates long discussions that are difficult to sum up. Although the diversity of the group can be challenging, the openness of the members creates the proper space for growth and interactions: "I remember being overwhelmed but also optimistic when I first joined the group [ ... ] I immediately thought I belong here! Yes, my disciplines differ from the others in the group [ ... ] I was happy that my perspective would be valued by many of the researchers" (Vignette 4).

The means of communication refers to two aspects that influence communication in the discussions in the group, namely language and understanding. Most of the group members agree that as the group started growing with the addition of late-joining $\mathrm{PhD}$ candidates, terminological differences as well as different "scientific languages" became more visible: "One had to start to explain oneself or rather realized that one was not understood and the different 'methodologies' became visible" (Vignette 7). At the beginning, this invoked criticism and made it difficult, especially for the new group members, to be understood while presenting their work in the group: "I felt my project was very criticized and not totally understood" (Vignette 3). In parallel, this very drawing of disciplinary boundaries also served as a starting point for the members to bridge the communication gap and making their research understandable to the others by explaining the terminology. This enabled open discussions regarding the disambiguation of terms used in individual projects, as well as constructive feedback: "There 'suddenly' were more open discussions and questions on concepts and theories, and different languages, e.g., factors, subjective, objective" (Vignette 7). Subsequently, group members from both sides of the spectrum (i.e., the "quantitatives" and the "qualitatives") started reducing language complexity, simplifying abstract concepts, and being more precise while explaining their work: "When I present my work in the mobil.LAB, I present it to friends, not to colleagues. I usually do not present complex mathematical problems. I focus on everyone's understanding of what I am doing and the significance and application of my research. I think the mobil.LAB is the perfect school to simplify a project, to make it communicable to everyone, and to get feedback from different perspectives" (Vignette 3). Despite the fact that there is no common scientific language in the group due to the disciplinary differences, the group has made an effort to develop a common communication language, which contributes to common understanding. 
Building on that, it can be argued that the use of language and the group's growth has influenced the collaboration among the members of the group. The collaboration defines the dynamics within the group. Our vignettes show that the majority of the group members feel that there is openness and respect for expressing their challenges as well as for constructive feedback. However, there have been occasions where the group's growth hampered collaboration. A good example for that is the failure of the monthly reading group as a platform for discussions. When it was set up, there were only five people who started their PhD project in quick succession, and were thus more open to discuss different literature not directly related to their developing individual research. In addition, four out of the five initial group members had similar research approaches. When the group grew, the diversity of the research approaches grew as well, which led to diverging research interests and eventually the demise of the reading group: "The disciplinary differences became more obvious when new members with more diverse approaches joined the group" (Vignette 5); “When we grew, our diversity grew in terms of our topical interests, disciplinary backgrounds, and how far along we each were in our research" (Vignette 2).

Another aspect of collaboration is the absence of competition between the members of the group: "That everyone is focusing solely on their own research topics and projects (all independent work), all with the same funding (no competition), and all with different timelines and schedules (no race to the finish line), supports a level of collegiality, as we are not in direct competition with each other" (Vignette 1). This cultivates an atmosphere of collegiality in the group. Although the group has worked successfully together in organizing events, such as conferences, workshops, and PhD courses, other than in the present article, the group has not conducted collaborative research so far.

The interactions in the group lead to consequences related to personal development, reflection, changes in the group-individual relationship, and conflict. These consequences were distributed across two distinct processes (i.e., productive and negotiation processes), which are described in the following sections.

\subsection{Productive Process}

The interactions function as reflexive exercises, where putting ourselves in other people's positions helps us to better understand our own issues and challenges: "The discussion in the group makes me reflect on my own process and I instantly feel more positively [sic] about my own work as I hear them reflect on processes and tasks that I am also facing" (Vignette 6). For some members, this open and outspoken reflection helps release stress and deal with mental blocks. Furthermore, viewing the group as a support network, many members mentioned that they trust the group enough to allow themselves to be exposed and be vulnerable, in order to present their problems and unfinished work. For some more than others, the group made them see aspects in their research that they would not pay attention to on their own: "After the mobil.LAB feedback, I started giving higher importance to the social approach, semantics, definitions, and terms in my research" (Vignette 3). Other important aspects of personal development are becoming a better writer through the exchange of written feedback and viewing one's research from a broader perspective. There have also been a lot of reflections on objectivity and subjectivity in research, with most of the members agreeing that there is no actual objectivity in research and there is always a degree of subjective interpretation at some stages of their research. Last but not least, most of the members agree that the interactions within the group sharpened their argumentation and their critical and political thinking.

These types of interactions had enablers: The above described development of friendship, but also the social life of the group, the common interest in sustainable mobility, the different stages of the $\mathrm{PhD}$ process, the imposed (earlier referred to as intended) set-up of the group, the colloquia, and the everyday working in a shared office. Interestingly, as indicated above, the vignettes suggest that the trigger as well as the means for these interactions was the fact that we were not speaking the same scientific language, wherefore we had to find a "common" language between our disciplines using the different languages as starting and reference points: "It forces me to be more precise, boil down 
abstract topics, and explain more clearly what I mean or what I do out of respect for the person doing different research" (Vignette 9); "I increasingly often have the impression that terminology became more important since the disciplinary boundaries became more obvious; however, I am not sure if this serves a clearer understanding for everybody or is the actual drawing of these boundaries" (Vignette 7). How the different use of language again interacts with the disciplinary origin was not reported by the authors, albeit an interesting aspect of interdisciplinary research and practice.

Through reflecting on our own personal and disciplinary perspectives and their relation to the other perspectives that are present within the group, every researcher started on a path of personal development. Within this production process, the issue of reflection is therefore a central element and vehicle for an occurring change on an individual level: "[The] kinds of happenings during group meetings, etc. clearly made me reflect and strengthen my view on my empirical data and reality in general" (Vignette 8). We term this development "productive" because the outcome of these types of interactions is substantially different from the starting point, e.g., an altered research and disciplinary perspective or a distinctively more political approach: "More specifically, we as a group are political, critical, and reflecting a lot. That has definitely influenced my individual project. My project got more political and focused on mobility politics" (Vignette 8); “My favorite part is when we give or receive some feedback, and we react as 'I would have never thought about this before'" (Vignette 3).

\subsection{Negotiation Process}

In contrast with the interactions within a productive process, interactions also took place as negotiation processes. These negotiation processes also took place in an additional spatiotemporal platform, which is the ceased reading group (see above). Additionally, some of the enablers of the productive process here acted as constraints, e.g., the stages of the $\mathrm{PhD}$ and the (mis)understanding across the group. This created a tension that sometimes resulted in conflict in the form of heated discussions, or even led to silencing and avoiding discussions completely.

The diverse disciplines and approaches often bring an extra level of conflict and misunderstanding, as well as frustration: "I find myself getting slightly annoyed with a question about why I am not asking the same questions in every interview and how I can then use the answers to conclude anything" (Vignette 6). There is also a tendency in the group to silence debates around diverse political opinions and worldviews: "For me, these discussions [ ... ] mainly made me express [my arguments] less in group contexts" (Vignette 7); "Social conflicts occur within the interdisciplinary dimension. Then, I feel I have to be careful to share my ideas or feedback" (Vignette 3). Despite the fact that the group shares similar interests and values in sustainable mobility, it does not mean that everyone has the same worldviews and political ideologies regarding how sustainable mobility should be achieved. These different worldviews have not been discussed extensively in the group so far.

We identified the negotiation process as taking place between the two poles of personal development and disciplinary gravity (the relative importance of one's own discipline). On the more common end, an interaction resulted in being taken back in by the discipline or approach one started from: "I am not carrying out a qualitative approach but [ ... ] consider it to enrich and support quantitative studies" (Vignette 3); "I often found myself thinking: 'OK, this is interesting and probably good to be exposed to, but I cannot use this'" (Vignette 2). On the other end of these interactions, there was again a form of personal development, but more focused on the individual in the form of acquiring a skill or gaining a competence: "I cannot say that the group had a big impact on the shaping of my epistemological foundation, but I can definitely recognize that it helped me with argumentation, sense-making, and communicating my research in a way that is understandable by different audiences" (Vignette 5).

Implicitly, these negotiations were about the extent to which the research group is a "team" sharing a common topic and research interest: Are we fulfilling the expectation from the set-up and proclamation of the mobil.LAB as an interdisciplinary research group? In contrast with the distinction that we set up between negotiation and productive processes, the negotiation and production of 
interdisciplinarity at times happen simultaneously, blurring the analytical boundaries drawn in the descriptive results. Furthermore, these processes are probably part of many researchers' personal development. However, they likely take place individually and in isolation, whereas the mobil.LAB made exploring "[ ... ] the different ontologies, epistemologies, and resulting methodologies and methods [ ... ] a very practical experience" (Vignette 7). Thus, according to our experience, this at least lays the ground for a more interdisciplinary understanding of research. This will be the focus of the discussion.

\subsection{Common Research Theme Evaded in the Vignettes}

The analysis of the vignettes revealed that members of the research group engaged mostly with the first part of the vignette prompt (influence on individual research) and not with the second, which was: "How does the interdisciplinary set-up of the mobil.LAB [ ... ] (re)shape the predefined common research theme of mobility culture?". Only a few vignettes referred directly or indirectly to this matter. Although there is a predefined common theme, every member works on her or his individual project. This observation, and the fact that most vignettes focused on the first part of the prompt, allows the conclusion that the common overarching theme of mobility cultures has little priority in the research process of the group.

\section{Discussion}

As presented in the Results section, the three main categories help us to understand how we practice interdisciplinarity in our research environment. Interactions serve as a carrier of both negotiation and productive processes. In this section, we will further discuss how the specific set-up of our doctoral research group where each researcher works on their own individual projects affects our interdisciplinary practices (understood colloquially). Furthermore, we reevaluate the usefulness of the separation into three categories and show that, in reality, our practice of interdisciplinarity is much more complex than this simple triptych. We also investigate the advantages and challenges associated with the three categories as they appeared in the autoethnographic vignettes. Furthermore, we link the analysis to the concept of intersubjectivity, which we consider as the key for the practice of interdisciplinarity in our doctoral research group. We understand intersubjectivity as the mutual sense-making of each other's research, background, and struggles. Intersubjectivity does not exist prior the group's interactions, but it rather exists as a potential, which is performed and enacted through the sharing of everyday experiential knowledge (e.g., perceptions, thoughts, linguistic meanings). We then take an additional interpretative step away from the initial results to relate these points to the current "ladder thinking" about different levels of interdisciplinarity (a ladder going from disciplinary to multidisciplinary to interdisciplinary to transdisciplinary); see [32] to question its validity as an applied concept.

\subsection{An Individual Set-Up: Multiple Individual Research and a Rigid Theoretical Framework}

As presented in the introduction, our research group was formed with the intent of producing an interdisciplinary sustainable mobility theory via individual dissertation projects without an overarching coordinated shared research project. This set-up is interdisciplinary in the sense that the researchers come from various multiple disciplines (see Figure 2 in the Methodology section) and are linked through a single theoretical framework. This has a direct impact on our interdisciplinary practices. For example, Vignette 9 states that "[ ... ] we are not really a 'team', we are not working on [a] joint project, but everyone is working on her or his individual project, and in the end, everyone is deciding about the direction on her or his own".

As it became obvious from the Results Section 4.4, none of our vignettes explicitly answered the question of "How does the interdisciplinary set-up of the mobil.LAB influence your individual research on mobility and (re)shape the predefined common research theme of mobility culture?"; therefore, it is hard to problematize this shaping mobility cultures. Each individual researcher has interpreted this 
imposed theme differently and "reshaped" it. As outlined below Section 5.5, several members of the group rejected it as the guiding concept for their research, either because it seemed too vague or too rigid to them, or because they questioned the application of the term "culture", which is in itself a very contested concept.

Although no agreement in the group exists on the "mobility cultures" as a research approach, there is an agreement on the normative approach - that current mobility regimes are unsustainable, and that the mobil.LAB commits to make mobilities more sustainable. Therefore, we posit that to enable the interdisciplinary practices, sustainable mobility as an overarching idea-in the form of a normative target-is preferable for the group's joint work. Hence, over time, the imposed theme was organically transformed into the theme of (un)sustainable mobility. What made sustainable mobility a rallying theme for our research group could be that, as opposed to mobility culture, sustainable mobility can be interpreted as a wicked problem, naturally calling for interdisciplinary collaboration in order to be solved, leading to the building of interlinkages between the disciplines. The guiding principle of sustainable mobility can be adapted and understood from the comfort of each researcher's disciplinary background while being interpreted differently by the others. While having become the overarching idea for the group, it has been variously taken up in the individual research projects. This is also owed to the different nature of each project; some are more "applied" and aim at results that can be directly translated into action and policy advice, while others are more exploratory and are targeted towards theory advances or being critical of the problem-solving function of (applied) research. This essentially means that in some individual research projects, mobility cultures are not taken up as a concept or idea.

\subsection{All Three Processes are Important and Can Act as Catalysts for Interdisciplinarity}

The separation between the interactions, negotiation processes, and productive processes that we operated in the Results section is only analytical, since both negotiation and productive processes are based on, and inextricably linked to, the interactions which can function as catalysts, or inhibitors, for interdisciplinary collaboration (see Figure 3). Furthermore, a single spatiotemporal platform like our monthly colloquia can be the stage for all three processes simultaneously. For example, one researcher found a catalyst to interdisciplinary research through a negotiation process in that they " [ ... ] learn to value different disciplinary perspectives and [ ... ] learn to understand quantitative, technology driven, problem/solution-oriented research" (Vignette 9). Another researcher mentions in their vignette that productive processes can be challenging for interdisciplinarity: "This openness is not a given and it requires a constant effort that we are not always able to accommodate, and in some cases, restraint and respect also play an important role, or at least a lack of them can derail the collaboration" (Vignette 4).

When analyzing the different sections of the vignettes categorized as interdisciplinary practices, we looked for passages describing advantages and challenges to directly answer our research question related to the different ways in which interdisciplinarity is embedded in daily practice, encompassing both advantages and challenges. We note that our three main analytical categories could be linked to challenges and advantages.

\subsubsection{Advantages}

The most mentioned advantage associated with interdisciplinary interactions is that they can lead one to reflect upon and improve research. They can also lead to problem solving, idea creation, and self-reflection. This can take the form of useful suggestions or open and constructive feedback. Both formal and informal discussions are included and can contribute to a change in perspective. Researchers consider it advantageous that their individual research can benefit from the outlook of other disciplines-for example, when it leads to adjusting one's argumentation or framing of the individual's research. We found that our interdisciplinary practices lead to improvements on research design. Finally, the researchers report a change of perspective and inspiration as a result. 


\subsubsection{Challenges}

The interdisciplinary practices of our research group also bring complex challenges. The main challenge reported in the vignettes related to the conflict avoidance strategy of quietly suppressing discussions on issues that are incommensurable. The following quote from Vignette 8 exemplifies this latent conflict and potential for misunderstanding: "[ . . ] one of our associated fellows telling me after a presentation about the production of space and different dimensions of space: 'Space means square meters for [sic] me.' [Expletive]! But yes, he is right! That is what it means for [sic] him". The vignettes likewise mention the challenge of dealing with different disciplinary languages which forces the expression of the obvious and the adaptation of the discourse to the diverse audience, requiring extra effort and added complexity. This necessitates an elaborate balancing act between self-trust and remaining open to feedback while being confronted by different understandings of normativity, the roles of researchers, and various epistemologies and ontologies.

Our interdisciplinary practices confronted the researchers with the diversity of perspectives. A certain feeling of insecurity can lead researchers to reproduce or even reinforce the disciplinary boundaries instead of crossing them as intended. The researchers reported finding it hard to step away from their disciplines and to find a balance between the simplicity of one's own discipline and the complexity of integrating ideas from another discipline. Interdisciplinarity is also fraught with the challenge of unconstructive feedback.

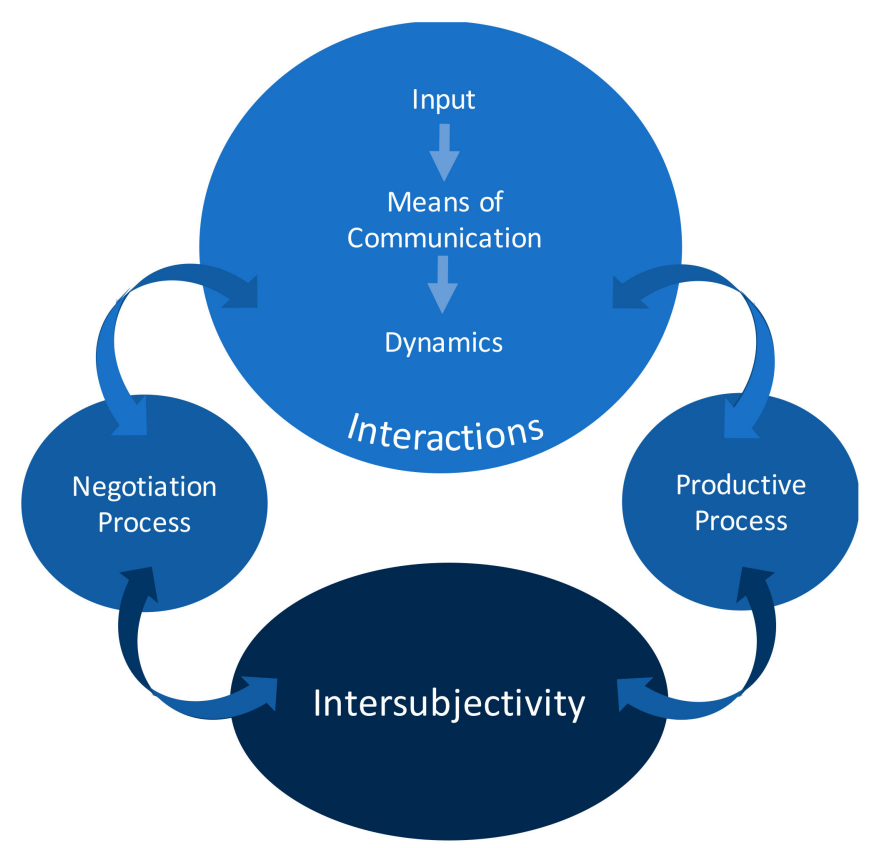

Figure 3. Interdisciplinary practices model.

Another known challenge to interdisciplinarity is that the work can create a new discipline in itself over time: An "interdiscipline" which becomes institutionalized [58]. As mentioned in the Introduction, mobilities research as such is the result of an interdisciplinary approach and could be characterized as an "interdiscipline". Schwanen's research on the interactions between Human Geography and research on sustainability transitions concludes that, while there are many benefits of interdisciplinary collaborations, there is also a risk of excluding or overly simplifying "cutting-edge thinking" within the discipline [58]. This risk is prevalent in the interdisciplinary practices described in this paper and within mobilities research as such; while being forced to simplify complex concepts is often viewed as a positive outcome of interdisciplinarity, it also has the risk of incomprehensible critique and plain misunderstanding, as exemplified by Schwanen as "the dreaded 'so what?' question" [58]. In the Results section, we have highlighted both positive (see Section 4.1 on Interdisciplinary Interactions) 
and negative interactions (see Section 4.3 on Negotiation Process) within the research group related to this. Many of the challenges of working in an interdisciplinary set-up can be ascribed to exactly this balance between oversimplifying and making oneself understood. While mobilities research has developed into an interdiscipline itself, within the mobil.LAB, the different disciplinary backgrounds still influence how each individual researcher interacts with this interdiscipline, as also showcased through Figure 2.

Interdisciplinarity appears as potentially destabilizing and requires one to have enough self-confidence and openness to others to allow themselves to be vulnerable. These attributes seem required for the productive processes to be in fact activated. Within our research "community", intersubjectivity has enabled and been enabled by interdisciplinary practices.

\subsection{Intersubjectivity Is the Key}

During our meeting to interpret the results together, the latent importance of intersubjectivity in our discussions became apparent, as it is performed through the productive and negotiation processes. We find that intersubjectivity is part of the infrastructure of interdisciplinarity (see Figure 3). In our understanding, intersubjectivity reflects the continuous effort that the individual researchers put into developing a mutual understanding of each other, transcending their own individual frames of perception and experiences. Our conceptualization of intersubjectivity is very much in line with the concept of intersubjectivity developed by Jürgen Habermas. The German philosopher states that "the aim of reaching understanding (Verständigung) is to bring about an agreement (Einverständnis) that terminates in the intersubjective mutuality of reciprocal comprehension, shared knowledge, mutual trust, and accord with one another" [59]. Habermas [59] uses the term intersubjectivity "[ ... ] to refer to the commonality established between subjects capable of speech and action by way of the understanding of identical meanings and the recognition of universal claims". Many of our vignettes mentioned the specific efforts that our group puts in place in order to ensure they can be understood by researchers from different disciplines.

The friendship and trust that we have developed in the few years since the start of our lab also enables this intersubjectivity: "[ ... ] the intersubjective commonality of a communicatively achieved agreement exists at the levels of normative accord, mutual trust in subjective sincerity [ ... ]" [59]. This is also in line with the thinking of Schutz [60], whose concept of intersubjectivity centers on the mutual understanding and equivalent status that friendship implies [61]. For example, Vignette 1 describes this feeling as "Similar to that of a family, we do not always agree on everything, but have opportunities for imaginative and lively discussions". Vignette 3 also portrays this friendship: "Once [an external] colleague asked me: 'How do you present your engineering work in the mobil.LAB?' [I answered] when I present my work in the mobil.LAB, I present it to friends, not to colleagues [ ... ] I think this social relationship is the glue that has helped us to open our minds to accept critique [sic] and feedback from different perspectives."

This intersubjectivity is not often mentioned in descriptions of interdisciplinarity in the literature, but it appears key to our practices. This intangible yet important aspect of interdisciplinary research is hard to quantify and does not easily fit with the "ladder" conception of interdisciplinarity.

\subsection{Against the Ladder, a Spectrum or Bottom-Up Approach to Understanding Interdisciplinarity in Practice}

Our collaborative autoethnographical experiment on the interdisciplinary practices of our doctoral research group which revealed the importance of intersubjectivity and the variety of different interdisciplinary interactions led us to question the usefulness of the hierarchical thinking about the various steps in the ladder of interdisciplinary research. Our group's evasion of the concept of mobility culture and the organic adoption of the problem of sustainable mobility instead leads us to agree with Thorén and Persson [62], who suggest an alternative "problem-feeding" conception of interdisciplinarity, which conceives interdisciplinary research as problem solving, as problems or solutions revealed in one discipline can be fed to another discipline for resolution and vice versa. 
Our vignettes show that we practice this problem feeding, especially during the PhD clinics. The authors call for adopting alternatives to the ladder conception of interdisciplinarity, especially in the case where there is no higher-level concept coordination, as is the case with the mobil.LAB.

Therefore, we propose adopting a bottom-up approach to interdisciplinarity, which would be defined by the practices that researchers consider interdisciplinary organized in a horizontal spectrum. This spectrum considers the various forms of integration between the disciplines, but is not hierarchically organized. For example, Lattuca [34], introduced in the literature review, proposes such a spectrum based on empirical material garnered from interviews with researchers from various disciplines involved in multidisciplinary research. The three main categories in our findings can be called upon to define this. Interdisciplinarity in practice is carried via interactions through negotiation processes and productive processes supported by intersubjectivity.

For interdisciplinarity to happen in practice, we conclude that having a combination not only of various disciplines, but also of backgrounds and ontologies, combined with a common "wicked" problem to solve can lead to productive "problem-based" interdisciplinarity. This raises the issue with current research funding practices, which are geared towards enabling this "ladder"-based interdisciplinarity.

\section{5. (Re)shaping the Predefined Common Research Theme}

To reflect on the lack of discussion of the common theme in the vignettes, it is necessary to include discussions within the group (before and after writing the vignettes) and to consider statements that indirectly refer to the "problem" of the common research theme.

Ever since the beginning of the second phase of the mobil.LAB, the "mobility cultures" approach has been met with very different and contradictory responses. Disciplinary background was one major factor for disagreement over validity of the approach. On the one hand, it was argued that "mobility cultures" provided a substantially widened set of factors that can be used for problem solving. On the other hand, substantial conceptual concerns were raised about engaging the term "culture". It is argued by some members that "culture" should be understood as a much more contested and multifaceted concept than it is in this approach, thereby also not fully embracing the paradigm shift of the mobilities turn.

At some point, a (tacit) agreement was found that "mobility cultures" is evaluated differently and is variously suitable for each member's individual topic. As one member puts it in his vignette (9), "because we are not working on a joint project, we do not need to convince each other regarding a certain standpoint or disciplinary perspective".

Several statements in the vignettes refer to the diversity of members within the research group, which makes it difficult to find a common language and to collaborate on a joint project. The vignette about the failure of the reading group is chosen as an example here for such a (small) joint project. It is stated: "[ ... ] Our failure to maintain the reading group is somehow reflective of our lack of interdisciplinarity. Because we had this open structure, we relied on individuals to select articles they thought or hoped would be interesting to the group as a whole. [ . . ] I do not feel like we succeeded [ ... ]" (Vignette 2). It becomes clear that to work with interdisciplinarity-for the benefit of each individual topic and for advancing the common research theme-a structured and targeted approach is necessary, e.g., a specific design for the reading group.

Furthermore, as several vignettes pointed out, it is also the different worldviews of members that spur disagreement in the group. One member highlights that this is the actual benefit (Vignette 9): "[... ] My opinion is that the diversity of worldviews in the group has a stronger impact on my research than the diversity of disciplines." In a tight-knit group like the mobil.LAB, differences in political leaning and worldviews cannot be fully omitted. However, from the standpoint of some members, they should not be omitted, but made productive (Vignettes 2 and 9). Naturally, personal and disciplinary backgrounds and choices reciprocally impact and reinforce ontological or political viewpoints. 
Members of the mobil.LAB have different opinions on how much these "wider" debates should shape the day-to-day research process of the group. One of the reasons for these different standpoints is highlighted in Vignette 7, paraphrased here: On the one hand, there is a need to increase complexity for theoretical advances, to achieve novel results, etc. On the other hand, there is a need to reduce complexity to make the results communicable and the process intersubjectively comprehensible. Within the group, priorities for one or the other sometimes clash; there are also disagreements over at which point in time either an increase or reduction of complexity is needed. Discussions on worldviews and ontologies, as well as about the common research theme of mobility culture, are therefore not always embraced by all members.

\section{Conclusions}

The findings from our autoethnographic analysis of our reflective vignettes highlight that the term "interdisciplinary" is initially no more than a descriptive label for the membership composition of the group. We conclude, based on our collaborative autoethnographic approach, that developing an interdisciplinary research environment requires embarking on a shared journey of reaching a higher level of intersubjectivity among the researchers through continuous interactions and discussions, developing productive partnerships, and negotiating conflicts. Therefore, this requirement for intersubjectivity frames our understanding of interdisciplinarity - in practice, first and foremost, as a communicative practice.

Fostering this intersubjective growth and creating an interdisciplinary environment is not a natural or simple task. As we posit, it would be true for any interdisciplinary research group that every researcher is more than their disciplinary background, ontological perspective, and axiomatic beliefs. A researcher's personal biography, prescribed to norms and values, political leanings, and previous experiences in research and practice guides their individual approach to researching the "wicked problem" and engaging with others in the group. We found that this diversity of opinion and expertise acts as both a facilitator and an impedance to the emergence of intersubjective understanding in the research group. The growth of the mobil.LAB over time has made achieving intersubjectivity more arduous, as the changing composition of the group members has required redeveloping the shared understandings and practices of communication. To form a basis for successful communication and collaboration, each individual must bring in a motivation to transcend subjectivity while maintaining identity and personal autonomy, a symmetry that Habermas calls the "idea of an intact intersubjectivity" [63]. Therefore, there is a certain agreement within the group to strive, as much as possible, for discursive practice free from domination.

While intersubjectivity is certainly needed for verification of research results, particularly when using qualitative methods, the vignettes have vividly demonstrated that the level of understanding, as well as the consensus reached, go far beyond the individual and collaborative research purposes. They also exceed the scope of interdisciplinarity. Doing collaborative and interdisciplinary research cannot be separated from relations of friendship, sharing similar values, and respecting each other's worldviews. Such a far-reaching encompassment of all aspects of life in the research process is close to Habermas's [64] understanding of the lifeworld at the intersection of objective, social, and subjective worlds. The lifeworld is essentially the "transcendental site where speaker and hearer meet, where they can reciprocally raise claims that their utterances fit the world" [64]. When practiced interdisciplinarity is essentially a continuous group effort to reach a higher level of mutual understanding, what are the consequences and implications? A focus on communicative practice challenges the output-oriented purpose of interdisciplinarity—that of "solving a wicked problem" —and instead suggests a shift towards appraisal of the practice of research collaboration itself as a process that is open-ended.

Intersubjectivity in research collaboration, as practiced in the mobil.LAB, has consequently been experienced as a liberating and emancipatory process by many members of the research group; it is unleashing creativity in research approaches and strengthening each member's ability to deconstruct 
and critique the socio-political foundations of the current unsustainable mobility regime. Such an open-ended process also invites revisiting the discussion of normativity in research, a debate held on numerous occasions within the research group. These discussions have made explicit to the group members that our individual research projects, as well as our group efforts, cannot be separated from our personal biographies, worldviews, and lives as (mobility) activists. This perception can be-and should be-engaged in a productive and transparent way to (re)politicize interdisciplinary research in general, and research on sustainability of mobility in particular.

As shown in the Introduction, the mobil.LAB is embedded into a larger "ecosystem" of actors and institutions researching sustainable mobilities. In exchange with the wider research community, the group's implicit set of rules-which is usually organizing the group's communication in the background-is made explicit, because in such exchanges, the limits of intersubjectivity become visible. In continuous interaction with other researchers, the members of the mobil.LAB are constantly reminded that the communicative consensus reached within the group is a fragile achievement. This once again highlights that successful cross-fertilization in interdisciplinary research communities starts with the communicative practice itself, irrespective of intended results.

The focus on research practice also allows a changing perspective on the theme of mobility cultures. The term culture, as used by Habermas, describes "[ . . ] a stock of knowledge from which participants in communication supply themselves with interpretations as they come to an understanding about something in the world" [64]. In the productive process based on intersubjectivity within the lifeworld, this stock of knowledge is also transformed, resulting in a new group consensus about the validity of knowledge [64]. Hence, research on mobility cultures should not be understood as an effort to identify "mobility culture(s)" in its entirety and complexity with the means of an interdisciplinary toolset. Intersubjective practice among mobility researchers should instead strive to reach a new consensus on the validity of knowledge that is needed in the transformation towards sustainable mobility. Consequently, we understand our collaborative research process as a way of doing mobility cultures.

Author Contributions: Each author contributed to the article in the following ways: Conceptualization, D.V., D.D.-R., A.F., J.M., M.M., L.N., E.S., and C.S.; methodology, D.V., D.D.-R., A.F., J.M., M.M., L.N., E.S., and C.S.; validation, D.V., D.D.-R., A.F., T.K., J.M., M.M., L.N., E.S., and C.S.; formal analysis, D.V., M.M., L.N., E.S., and C.S.; investigation, D.V., D.D.-R., A.F., T.K., J.M., M.M., L.N., E.S., and C.S.; data curation, D.V., M.M., L.N., and E.S.; writing-original draft preparation, D.V., D.D.-R., A.F., T.K., J.M., M.M., L.N., E.S., and C.S.; writing一review and editing, D.V., D.D.-R., A.F., T.K., J.M., M.M., L.N., E.S., and C.S.; visualization, D.D.-R., DV., A.F., and E.S.; supervision, D.V.; project administration, D.V., A.F., M.M., L.N., and C.S. All authors have read and agreed to the published version of the manuscript.

Funding: This work was supported by the German Research Foundation (DFG) and the Technical University of Munich (TUM) in the framework of the Open Access Publishing Program. The mobil.LAB doctoral research group is funded as Promotionskollege 032 by the Hans-Böckler-Foundation.

Acknowledgments: The authors would like to sincerely thank Gebhard Wulfhorst (Technical University of Munich) and Sven Kesselring (Nürtingen-Geislingen University), the co-speakers of the mobil.LAB, for their support of this work and the activities the related to the task. We are also grateful to the editors of the special issue, Henrike Rau and Joachim Scheiner, for their editorial guidance and support. We also thank the anonymous reviewers for their feedback and suggestions which enabled us to improve the paper.

Conflicts of Interest: The authors declare no conflict of interest. The funders had no role in the design of the study, in the collection, analyses, or interpretation of data, in the writing of the manuscript, or in the decision to publish the results.

\section{References}

1. Banister, D. The Sustainable Mobility Paradigm. Transp. Policy 2008, 15, 73-80. [CrossRef]

2. Givoni, M.; Banister, D. (Eds.) Moving Towards Low Carbon Mobility; Edward Elgar Publishing: Cheltenham, UK, 2013; ISBN 978-1-78100-722-8.

3. Hopkins, D.; Higham, J. (Eds.) Low Carbon Mobility Transitions; Goodfellow Publishers: Oxford, UK, 2016; ISBN 978-1-910158-65-4.

4. Sheller, M. Mobility Justice: The Politics of Movement in an Age of Extremes; Verso: London, UK, 2018; ISBN 978-1-78873-092-1. 
5. Rittel, H.W.J.; Webber, M.M. Dilemmas in a General Theory of Planning. Policy Sci. 1973, 4, $155-169$. [CrossRef]

6. Funtowicz, S.O.; Ravetz, J.R. A New Scientific Methodology for Global Environmental Issues. In Ecological Economics: The Science and Management of Sustainability; Costanza, R., Ed.; Columbia University Press: New York, NY, USA, 1991; pp. 137-152. ISBN 978-0-231-07563-3.

7. Funtowicz, S.O.; Ravetz, J.R. Science for the Post-Normal Age. Futures 1993, 25, 739-755. [CrossRef]

8. Nowotny, H.; Scott, P.; Gibbons, M. Re-Thinking Science: Knowledge and the Public in an Age of Uncertainty; Polity Press: Cambridge, MA, USA, 2001; ISBN 978-0-7456-2607-9.

9. Rau, H.; Fahy, F. Introduction: Sustainability Research in the Social Sciences-Concepts, Methodologies and the Challenge of Interdisciplinarity. In Methods of Sustainability Research in the Social Sciences; Fahy, F., Rau, H., Eds.; Sage Publications: London, UK, 2013; pp. 3-24. ISBN 978-0-85702-521-0.

10. Rau, H.; Goggins, G.; Fahy, F. From invisibility to Impact: Recognising the Scientific and Societal Relevance of Interdisciplinary Sustainability Research. Res. Policy 2018, 47, 266-276. [CrossRef]

11. Sheller, M.; Urry, J. The New Mobilities Paradigm. Environ. Plan. A 2006, 38, 207-226. [CrossRef]

12. Urry, J. Mobilities; Polity Press: Cambridge, UK; Malden, MA, USA, 2007; ISBN 978-0-7456-3418-0.

13. Urry, J. Climate Change and Society; Michie, J., Ed.; Polity Press: Cambridge, UK; Malden, MA, USA, 2011; ISBN 978-0-7456-5036-4.

14. Wilde, M.; Klinger, T. Integrierte Mobilitäts-und Verkehrsforschung: Zwischen Lebenspraxis und Planungspraxis. In Verkehr Und Mobilität Zwischen Alltagspraxis Und Planungstheorie; Wilde, M., Gather, M., Neiberger, C., Scheiner, J., Eds.; Springer Fachmedien Wiesbaden: Wiesbaden, Germany, 2017; pp. 5-23. ISBN 978-3-658-13700-7.

15. Urry, J. Sociology Beyond Societies: Mobilities for the Twenty-First Century; Routledge: London, UK; New York, NY, USA, 2000; ISBN 978-0-415-19088-6.

16. Adey, P.; Bissell, D.; Hannam, K.; Merriam, P.; Sheller, M. (Eds.) The Routledge Handbook of Mobilities; Routledge, Taylor \& Francis Group: London, UK, 2014; ISBN 978-0-415-66771-5.

17. Freudendal-Pedersen, M.; Hartmann-Petersen, K.; Kjærulff, A.A.; Nielsen, L.D. Interactive Environmental Planning: Creating Utopias and Storylines within a Mobilities Planning Project. J. Environ. Plan. Manag. 2017, 60, 941-958. [CrossRef]

18. Freudendal-Pedersen, M.; Kesselring, S. (Eds.) Exploring Networked Urban Mobilities; Networked Urban Mobilities Series; Routledge: New York, NY, USA, 2017.

19. Rau, H. (Im)mobility and Environment-Society Relations: Arguments for and Against the 'Mobilisation' of Environmental Sociology. In Environmental Sociology; Gross, M., Heinrichs, H., Eds.; Springer: Dordrecht, The Netherlands, 2010; pp. 237-254. ISBN 978-90-481-8729-4.

20. Rau, H. Das neue Mobilitätsparadigma als Regulierungsansatz für die Wechselbeziehungen von Umwelt, Politik und Gesellschaft. In Natur Und Gesellschaft: Gestaltung Und Regulation Der Natur-Gesellschaftsbeziehungen; Gesowip: Basel, Switzerland, 2011; pp. 93-128.

21. Stehr, N.; Weingart, P. (Eds.) Practising Interdisciplinarity; University of Toronto Press: Toronto, ON, Canada; Buffalo, NY, USA, 2000; ISBN 978-0-8020-4328-3.

22. Maasen, S. Inducing Interdisciplinarity: Irresistible Infliction? The Example of a Research Group at the Center for Interdisciplinary Research (ZiF), Bielefeld, Germany. In Practising Interdisciplinarity; Stehr, N., Weingart, P., Eds.; University of Toronto Press: Toronto, ON, Canada, 2000; pp. 173-193. ISBN 978-1-4426-7872-9.

23. Kesselring, S.; Wulfhorst, G. mobil.LAB: Sustainable Mobility in the Munich Metropolitan Area-Designing Mobility Cultures. Available online: https://www.bgu.tum.de/fileadmin/w00blj/sv/PDF/mobil.LAB_pdf_ files/mobil.LAB-Concept.pdf (accessed on 10 May 2019).

24. Deffner, J.; Götz, S.; Schubert, S.; Potting, C.; Stete, G.; Tschann, A.; Loose, W. Schlussbericht Zu Dem Projekt "Nachhaltige Mobilitätskultur": Entwicklung Eines Integrierten Konzepts Der Planung, Kommunikation Und Implementierung Einer Nachhaltigen, Multioptionalen Mobilitätskultur; Institut für Sozial-ÖkologischeForschung (ISOE): Frankfurt am Main, Germany, 2006.

25. Wulfhorst, G.; Kesselring, S. Perspectives on Sustainable Mobility in Metropolitan Regions: Shaping Mobility Cultures. In Sustainable Mobility in Metropolitan Regions; Klug, S., Ed.; Springer Fachmedien Wiesbaden: Wiesbaden, Germany, 2016; pp. 201-221. ISBN 978-3-658-14427-2. 
26. Klein, J.T. Crossing Boundaries: Knowledge, Disciplinarities, and Interdisciplinarities; University Press of Virginia: Charlottesville, VA, USA, 1996; ISBN 978-0-8139-1678-1.

27. Castán Broto, V.; Gislason, M.; Ehlers, M.-H. Practising interdisciplinarity in the interplay between disciplines: Experiences of established researchers. Environ. Sci. Policy 2009, 12, 922-933. [CrossRef]

28. Scerri, E.R. Interdisciplinary Research at the Caltech BeckmanInstitute. In Practising Interdisciplinarity; Stehr, N., Weingart, P., Eds.; University of Toronto Press: Toronto, ON, Canada, 2000; pp. 194-214. ISBN 978-0-8020-4328-3.

29. Van Noorden, R. Interdisciplinary research by the numbers. Nat. News 2015, 525, 306. [CrossRef]

30. Banister, D.; Stead, D.; Steen, P.; Akerman, J.; Dreborg, K.; Nijkamp, P.; Schleicher-Tappeser, R. (Eds.) Transport, development and sustainability. In European Transport Policy and Sustainable Mobility; Spon Press: London, UK, 2000; ISBN 978-0-415-23189-3.

31. Aboelela, S.W.; Larson, E.; Bakken, S.; Carrasquillo, O.; Formicola, A.; Glied, S.A.; Haas, J.; Gebbie, K.M. Defining Interdisciplinary Research: Conclusions from a Critical Review of the Literature. Health Serv. Res. 2007, 42, 329-346. [CrossRef]

32. Organisation for Economic Cooperation and Development. Interdisciplinarity: Problems of Teaching and Research in Universities; Center for Educational Research and Innovation: Paris, France, 1972; p. 307.

33. Rosenfield, P.L. The potential of transdisciplinary research for sustaining and extending linkages between the health and social sciences. Soc. Sci. Med. 1992, 35, 1343-1357. [CrossRef]

34. Lattuca, L.R. Creating Interdisciplinarity: Grounded Definitions from College and University Faculty. Hist. Intellect. Cult. 2003, 3, 20.

35. Klinger, T.; Lanzendorf, M. Moving between Mobility Cultures: What Affects the Travel Behavior of New Residents? Transportation 2016, 43, 243-271. [CrossRef]

36. Klinger, T.; Kenworthy, J.R.; Lanzendorf, M. Dimensions of Urban Mobility Cultures: A Comparison of German Cities. J. Transp. Geogr. 2013, 31, 18-29. [CrossRef]

37. Götz, K.; Deffner, J.; Klinger, T. Mobilitätsstile und Mobilitätskulturen- Erklärungspotentiale, Rezeption und Kritik. In Handbuch Verkehrspolitik; Schwedes, O., Canzler, W., Knie, A., Eds.; Springer Fachmedien Wiesbaden: Wiesbaden, Germany, 2016; pp. 781-804. ISBN 978-3-658-04692-7.

38. Klinger, T. Städtische Mobilitätskulturen Und Wohnumzüge; Springer Fachmedien Wiesbaden: Wiesbaden, Germany, 2017; ISBN 978-3-658-17230-5.

39. Wulfhorst, G.; Kenworthy, J.; Kesselring, S.; Kuhnimhof, T.; Lanzendorf, M.; Priester, R. Mobility Cultures in Megacities: Resulting from a Global Study. In Proceedings of the 13th World Conference on Transportation Research, Rio de Janeiro, Brazil, 15-18 July 2013; p. 20.

40. Ellis, C.; Adams, T.E.; Bochner, A.P. Autoethnography: An Overview. Hist. Soc. Res. 2011, 36, $273-290$.

41. Holman Jones, S.L.; Adams, T.E.; Ellis, C. Handbook of Autoethnography, 2nd ed.; Routledge: Oxon, UK, 2016; ISBN 978-1-59874-600-6.

42. Alvesson, M.; Sköldberg, K. Reflexive Methodology: New Vistas for Qualitative Research; Sage Publications: London, UK; Thousand Oaks, CA, USA; New Delhi, India, 2000; ISBN 0-8039-7707-7.

43. Chang, H.; Ngunjiri, F.W.; Hernandez, K.-A.C. Collaborative Autoethnography; Left Coast Press: Walnut Creek, CA, USA, 2013; ISBN 978-1-59874-555-9.

44. Anderson, L. Analytic Autoethnography. J. Contemp. Ethnogr. 2006, 35, 373-395. [CrossRef]

45. Pitard, J. Using Vignettes Within Autoethnography to Explore Layers of Cross-Cultural Awareness as a Teacher. Forum Qual. Soc. Res. 2016, 17, 17.

46. Humphreys, M. Getting Personal: Reflexivity and Autoethnographic Vignettes. Qual. Inq. 2005, 11, 840-860. [CrossRef]

47. Murakami-Ramalho, E.; Piert, J.; Militello, M. The Wanderer, the Chameleon, and the Warrior: Experiences of Doctoral Students of Color Developing a Research Identity in Educational Administration. Qual. Inq. 2008, 14, 806-834. [CrossRef]

48. Moore, J.; Scarduzio, J.A.; Plump, B.; Geist-Martin, P. The Light and Shadow of Feminist Research Mentorship: A Collaborative Autoethnography of Faculty-Student Research. J. Res. Pract. 2013, 9, M8.

49. Gurvitch, R.; Carson, R.L.; Beale, A. Being a protégé: An autoethnographic view of three teacher education doctoral programs. Mentor. Tutor. Partnersh. Learn. 2008, 16, 246-262. [CrossRef]

50. Hernández, F.; Sancho, J.M.; Creus, A.; Montané, A. Becoming University Scholars: Inside Professional Autoethnographies. J. Res. Pract. 2010, 6, 15. 
51. Bracken, L.J.; Oughton, E.A. "What do you mean?" The importance of language in developing interdisciplinary research. Trans. Inst. Br. Geog. 2006, 31, 371-382. [CrossRef]

52. Bissell, D.; Vannini, P.; Jensen, O.B. Intensities of mobility: Kinetic energy, commotion and qualities of supercommuting. Mobilities 2017, 12, 795-812. [CrossRef]

53. Patton, M.Q. Qualitative Evaluation and Research Methods; Sage Publications: Newbury Park, CA, USA, 1990; ISBN 978-0-8039-3779-6.

54. Ryan, G.W.; Bernard, H.R. Techniques to Identify Themes. Field Methods 2003, 15, 85-109. [CrossRef]

55. Lincoln, Y.S.; Guba, E.G. Naturalistic Inquiry; Sage Publications: Beverly Hills, CA, USA, 1985; ISBN 978-0-8039-2431-4.

56. Barbour, R.S. Quality of Data Analysis. In The SAGE Handbook of Qualitative Data Analysis; Flick, U., Ed.; Sage Publications: London, UK, 2014; ISBN 978-1-4462-0898-4.

57. Gibbs, G.R. Using Software in Qualitative Analysis. In The SAGE Handbook of Qualitative Data Analysis; Sage Publications: London, UK, 2014; pp. 277-294. ISBN 978-1-4462-0898-4.

58. Schwanen, T. Thinking complex interconnections: Transition, nexus and Geography. Trans. Inst. Br. Geogr. 2018, 43, 262-283. [CrossRef]

59. Habermas, J. On the Pragmatics of Communication; Cooke, M., Ed.; Studies in Contemporary German Social Thought; MIT Press: Cambridge, MA, USA, 1998; ISBN 978-0-262-08265-5.

60. Schutz, A. The Phenomenology of the Social World; Northwestern University Press: Evanston, IL, USA, 1967; ISBN 0-8101-0390-7.

61. Crepeau, E.B. Achieving Intersubjective Understanding: Examples from an Occupational Therapy Treatment Session. Am. J. Occup. Ther. 1991, 45, 1016-1025. [CrossRef]

62. Thorén, H.; Persson, J. The Philosophy of Interdisciplinarity: Sustainability Science and Problem-Feeding. J. Gen. Philos. Sci. 2013, 44, 337-355. [CrossRef]

63. Habermas, J. Studies in Contemporary German Social Thought. In Postmetaphysical Thinking: Philosophical Essays; MIT Press: Cambridge, MA, USA, 1992; ISBN 978-0-262-08209-9.

64. Habermas, J. The Theory of Communicative Action: Volume 2. Lifeworld and System: A Critique of Functionalitst Reason; Beacon Press: Boston, MA, USA, 1987; Volume 2, ISBN 0-8070-1400-1. 